\title{
Jeans des rues
}

La photographie à l'épreuve du jean

Street Jeans

Fleur Beauvieux, Justine Blanckaert et Claudia Coppola

\section{(2) OpenEdition}

Journals

Édition électronique

URL : https://journals.openedition.org/tc/4905

DOI : $10.4000 /$ tc. 4905

ISSN : 1952-420X

Éditeur

Éditions de l'EHESS

Édition imprimée

Date de publication : 1 décembre 2009

Pagination : 378-387

ISBN : 978-2-7351-1301-9

ISSN : 0248-6016

Référence électronique

Fleur Beauvieux, Justine Blanckaert et Claudia Coppola, « Jeans des rues », Techniques \& Culture [En ligne], 52-53 | 2009, mis en ligne le 01 août 2010, consulté le 29 septembre 2022. URL : http:// journals.openedition.org/tc/4905; DOI : https://doi.org/10.4000/tc.4905 


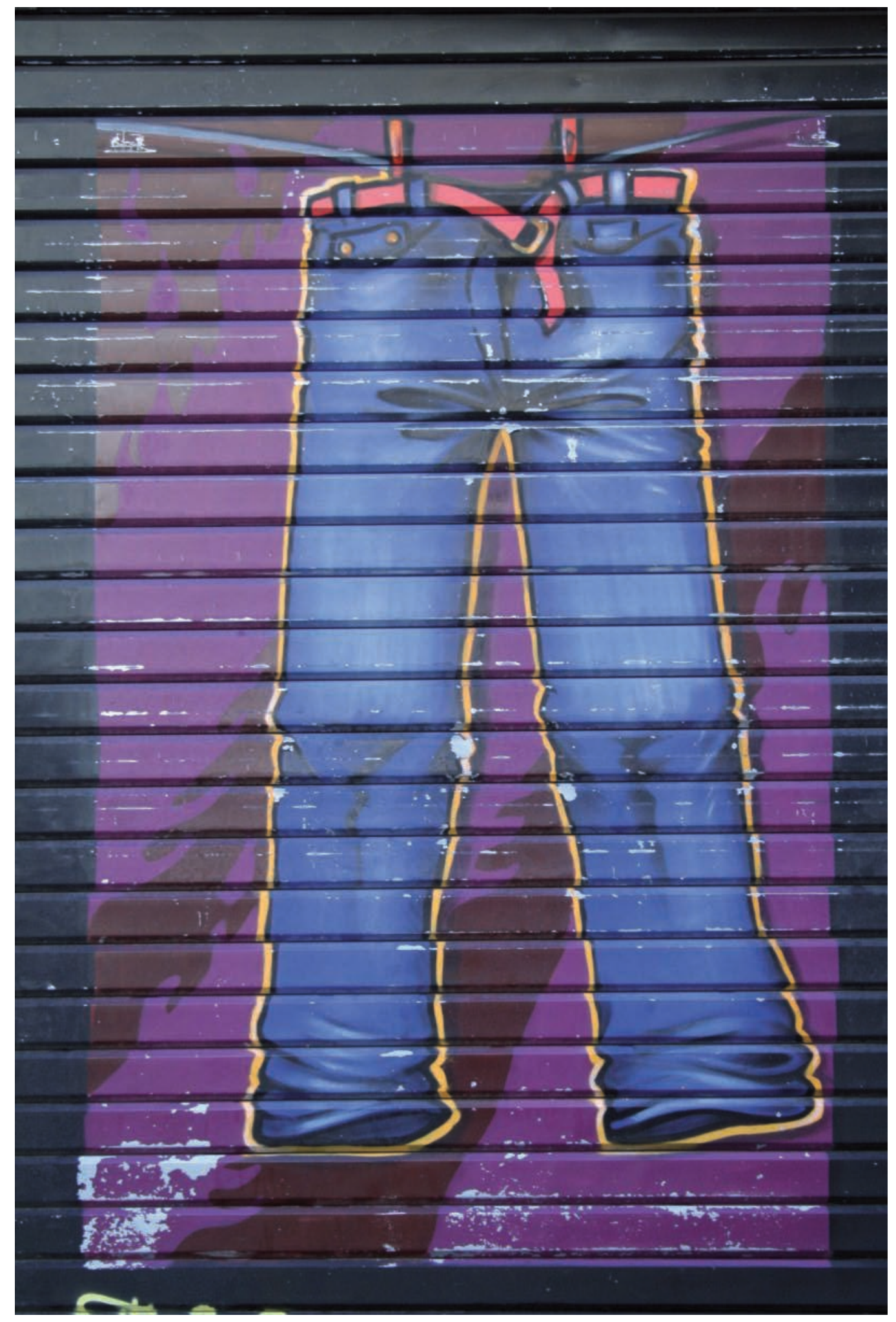




\section{Curiosa}

Fleur Beauvieux, Justine Blanckaert

\& Claudia Coppola

Technologies

EHESS Marseille

fleurbvx@hotmail.fr ; justineblanckaert@hotmail.fr

\& claudia_coppola2@yahoo.it

\section{JEANS DES RUES}

\section{La photographie à l'épreuve du jean}

La série d'images présentées ici a été conçue tout à la fois comme un exercice inédit de terrain, une réflexion et une enquête collective menée dans le cadre d'une recherche plus large sur l'utilisation de la photographie comme instrument heuristique adapté aux sciences sociales et l'invite à dépasser l'espace trop contraint de l'illustration de terrain.

En mettant la photographie au service de nos expérimentations réflexives et méthodiques, nous avons élaboré un travail collectif ${ }^{1}$ fondé sur la construction d'un micro-terrain avec comme point de départ, dans le cas présent, l'article de Daniel Miller, présenté dans ce Thema, « Le Bluejean. Pourquoi la technique vient en dernier » que nous avons matérialisé et mis à l'épreuve, en images.

Comment dépasser un travail illustratif ? Comment faire nôtre la démarche analytique et explicative de l'auteur, l'éprouver dans la rue avec une technique (la photographie numérique) tout à la fois nouvelle et largement disséminée ? Comprenons-nous bien, la photographie n'est pas nouvelle dans les sciences sociales, mais son utilisation a été longtemps cantonnée à la seule fonction illustrative. Ici, nous avons essayé de mobiliser les concepts de cadrage, de champ, de distance, d'angle, de lumière, etc. dans un travail de micro-ethnographie de terrain. La photographie peut jouer une médiation entre théorie abstraite et chose écrite. Par la figuration et la mise en images, elle permet la description fine. De même, par l'analyse des subjectivités en présence, celle des représentés, celle du photographe, elle permet de générer un nouvel espace d'objectivation. La position des photographes serait alors (si nous en avions la latitude) la première chose à décrire; elle est princeps à la démarche scientifique. 
Dans son article sur les jeans, Daniel Miller, se fondant sur les pratiques et sur les usages des jeans, nous invite à une observation subtile et raisonnée. Il parle de ses terrains, notamment dans les rues de Londres où il a interviewé des personnes portant des jeans. Dans le temps bref qui nous était imparti, nous avons rebondi sur son approche et sommes allées plus loin dans une démarche active et constructiviste en sollicitant les passants dans le centre-ville de Marseille et en les interrogeant à propos de leurs jeans. Nous leur avons demandé s'ils accepteraient de poser avec leurs jeans et de choisir un mot qui illustrerait cette représentation, leur relation aux jeans, à leur jean, ce jour-là.

En mouvement, nous avons improvisé des prises de vue et chaque personne était libre de jouer le jeu à sa façon.

Nous souhaitions en outre observer cette mise en scène personnelle. Cela constituait l'expérience photographique spontanément mise en place. Les cadrages ne variaient guère, et, dans un souci de respect du droit à l'image, incontournable dans la photographie contemporaine, nous n'avons pas photographié les visages.

Dans ce dispositif quasi expérimental, mais éphémère et en situation « naturelle »- le temps d'un entretien et de sa validation par l'image d'un mot et d'un objet - l'individu photographié est acteur, il se met en scène. Il élabore sa représentation à travers sa posture et l'expression qu'il y attache. C'est sa marge de manœuvre, le « jeu » entre eux, nous et le reste de la ville que nos photographies tentent d'objectiver. Les acteurs se montrent, s'exposent et caractérisent un lien à un objet, tout à la fois intime et public.

Les photos qui suivent sont les reflets d'une opération pragmatique originale et d'une esthétique ordinaire. Elles constituent une esquisse de réponse à notre questionnement expérimental sur une des façons d'utiliser la photo en ethnologie. À ce titre, elles sont les données empiriques « coproduites », par les personnes interrogées et nous-mêmes. Elles sont les matériaux bruts (nous en avons sélectionné un nombre très réduit) et nous les avons disposés afin de créer une narration imagée qui, nous l'espérons, peut également ouvrir un espace d'interprétations multiples.

\section{NOTES}

Toutes les photos sont des auteurs, sauf mention contraire.

1. Séminaire «Pratiques photographiques» 2008-2010, F. Joulian de l'EHESS Marseille. <http://centre-norbertelias.ehess.fr/document.php?id=682> 


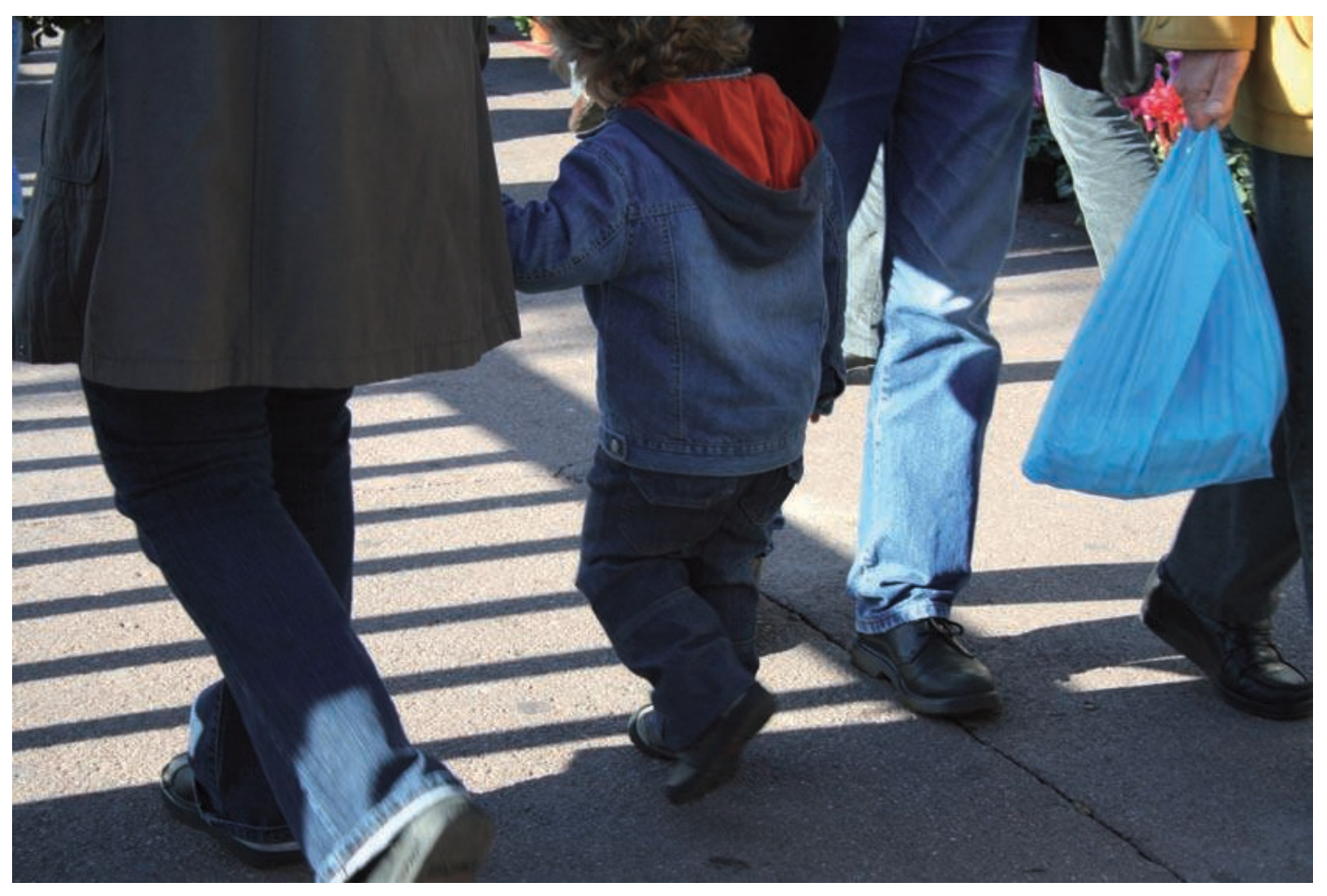




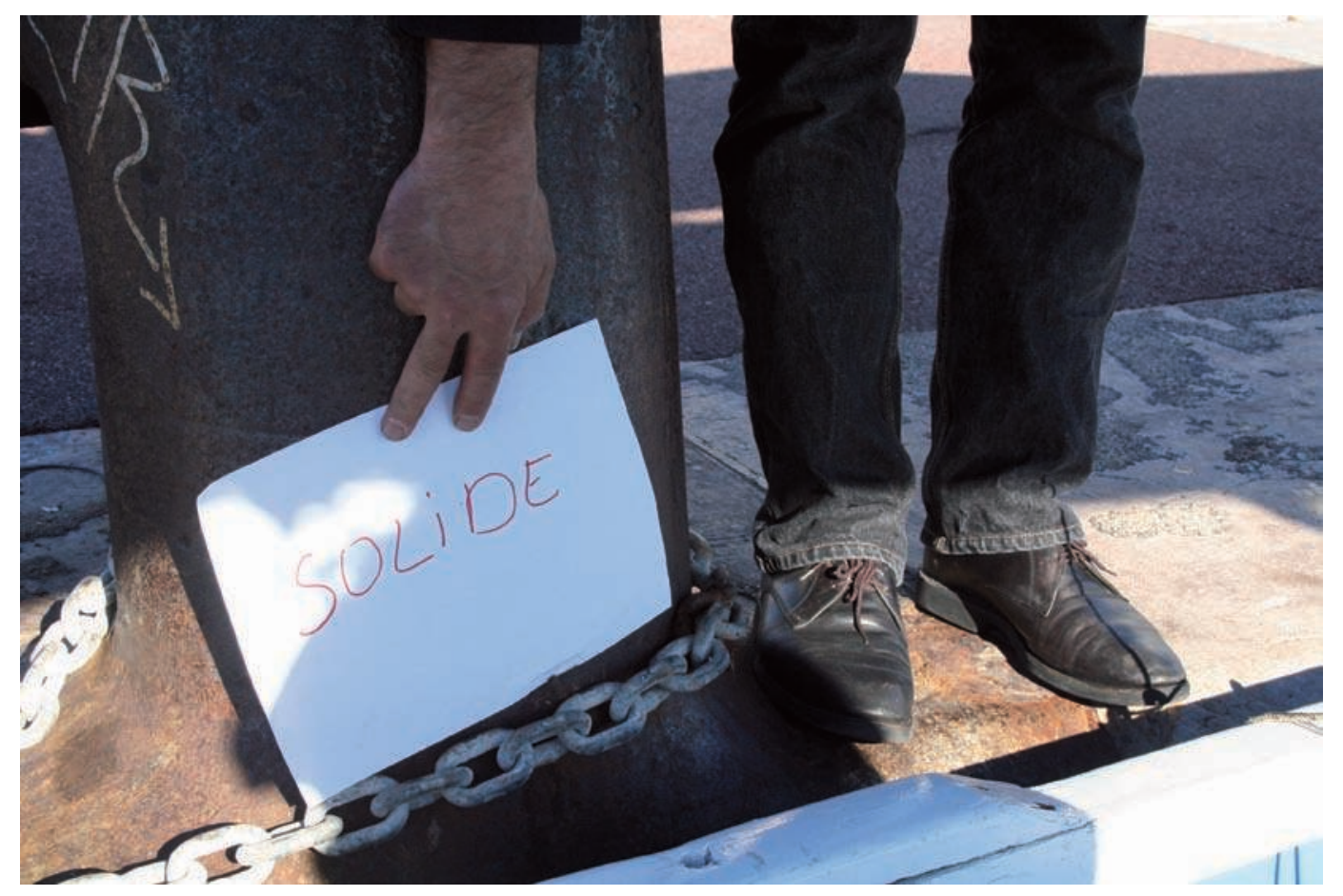



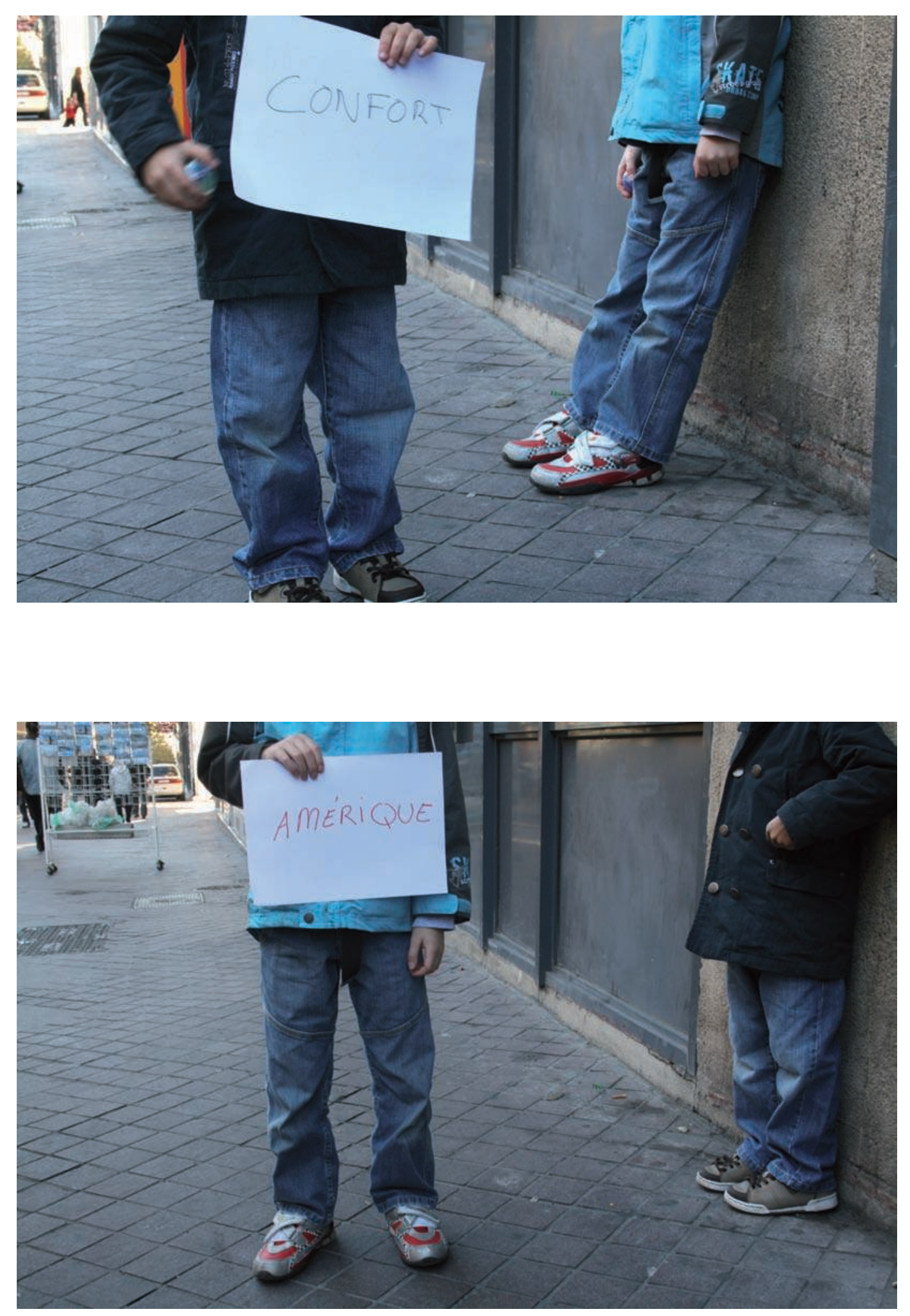

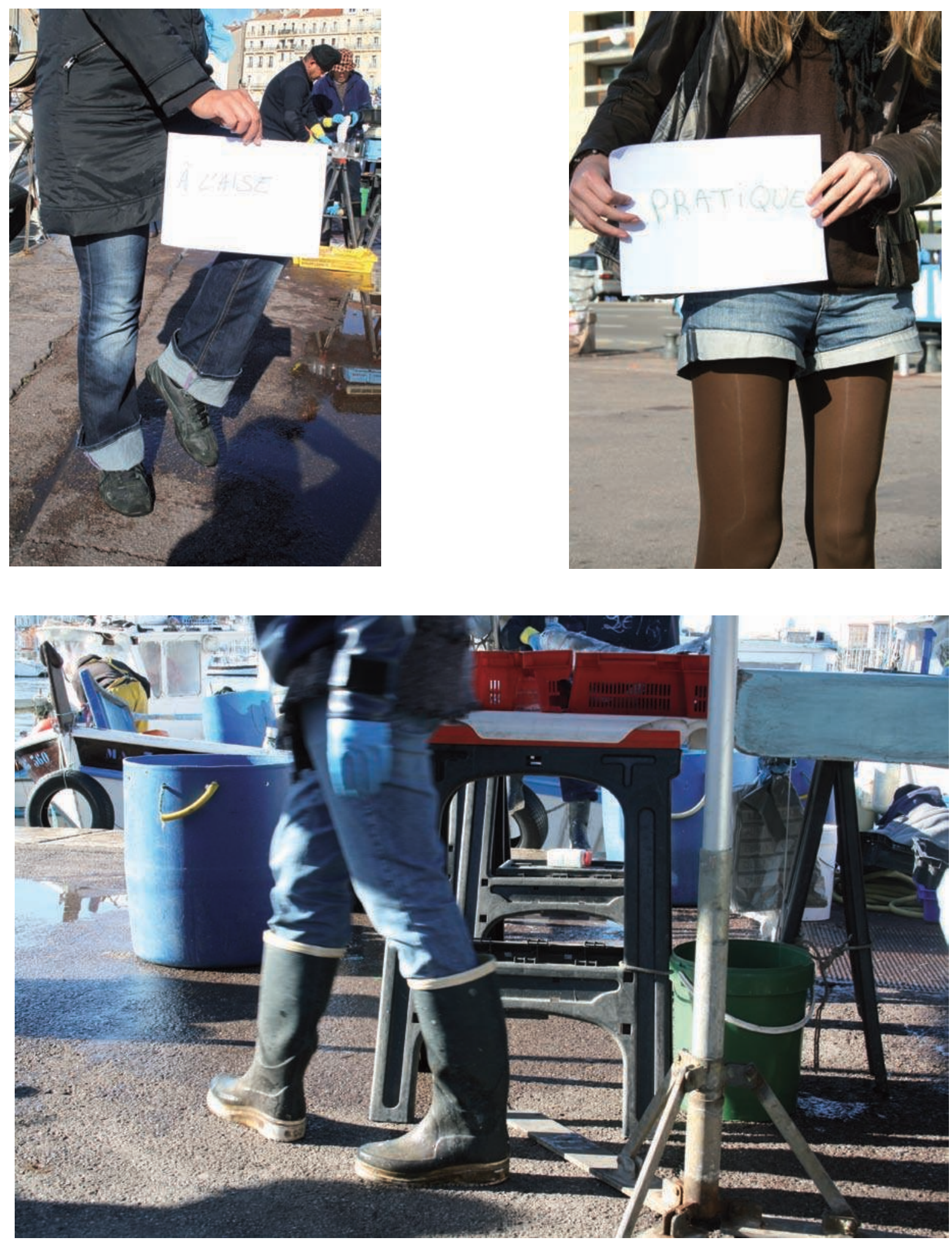

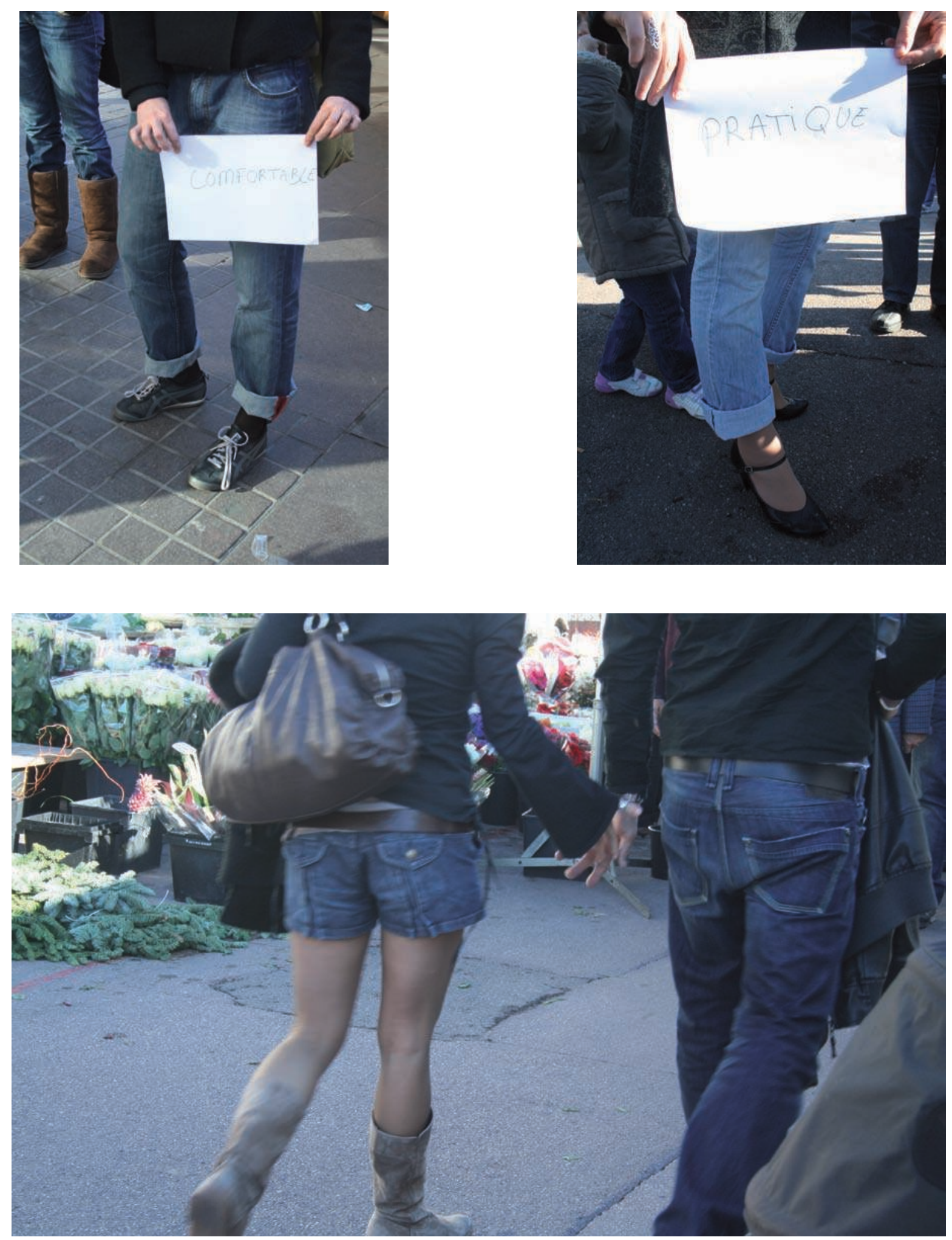

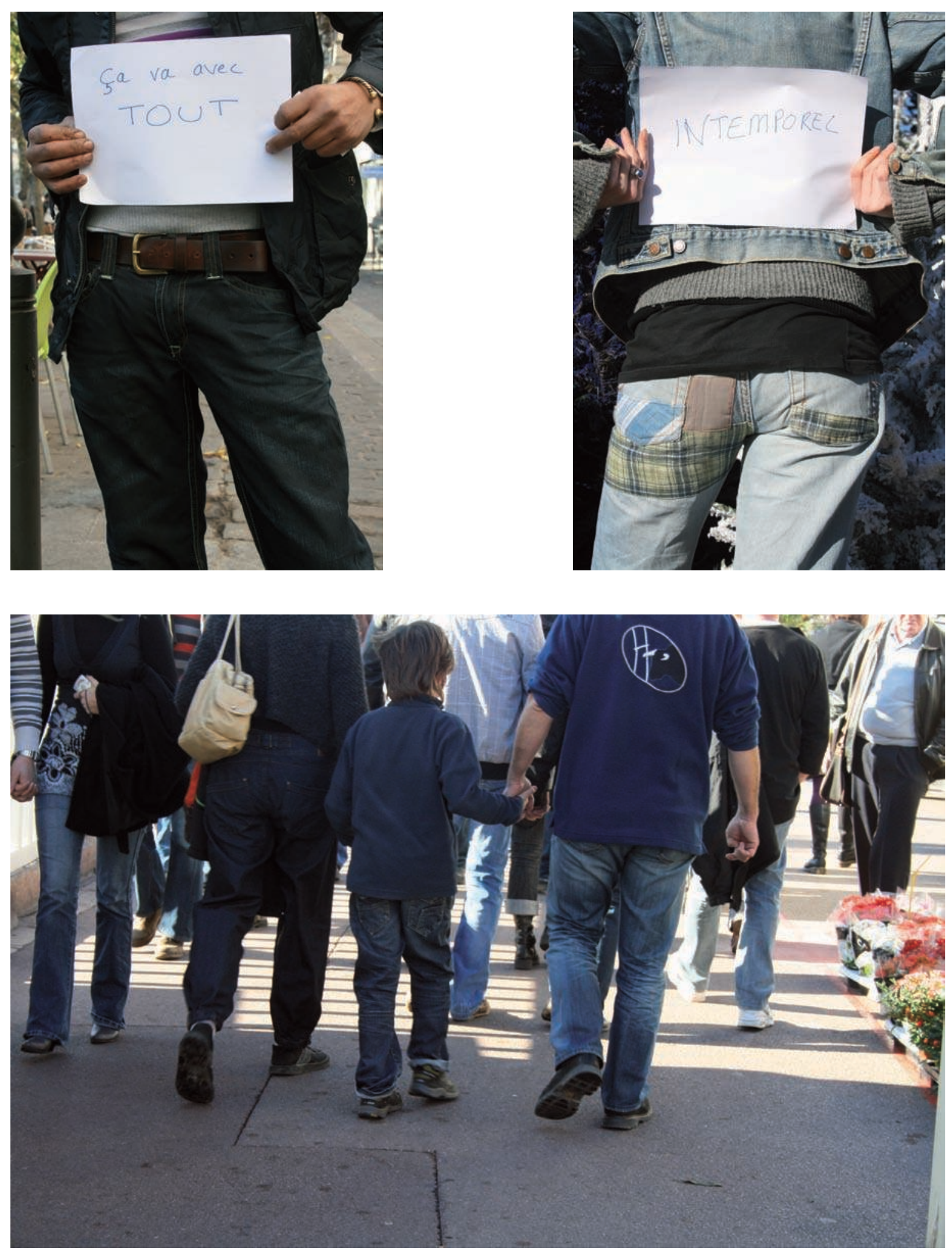

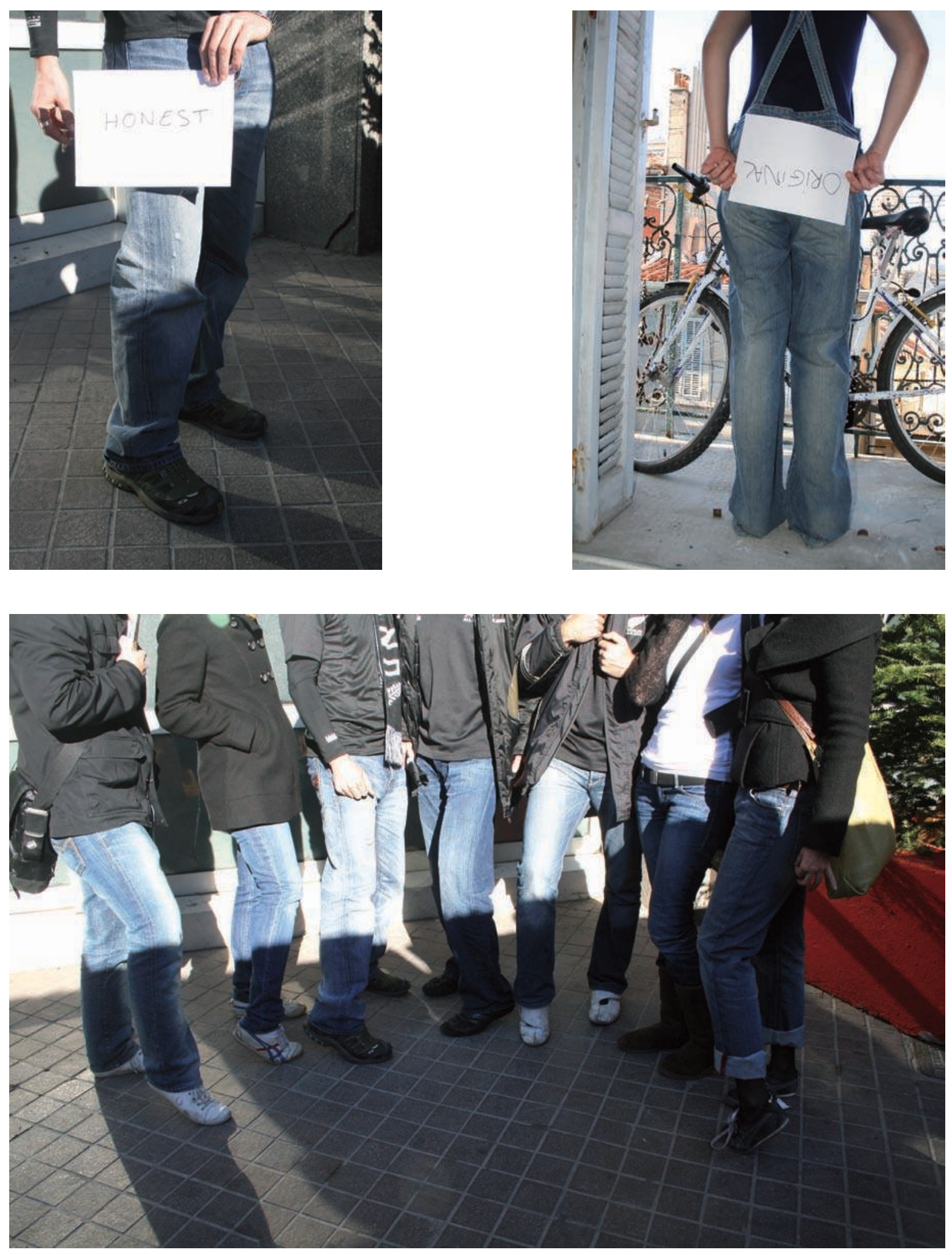